\title{
Gerenciamento de rejeitos radioativos da iodoterapia
}

\author{
André R. M. Silva ${ }^{\mathrm{a}}$ e Helena C. Santos ${ }^{\mathrm{a}}$ \\ ${ }^{a}$ Instituto Federal de Educação, Ciência e Tecnologia de Pernambuco (IFPE), 50740-540, Recife - PE, Brasil \\ andrerichard88@bol.com.br
}

\section{RESUMO}

A utilização de radioisótopos no tratamento de diversas patologias no serviço de medicina nuclear, principalmente na iodoterapia, requer um conhecimento especializado quanto à deposição dos rejeitos radioativos que são gerados durante o internamento do paciente. É necessário seguir o protocolo de segregação e armazenagem, visando seguir as normas de proteção radiológica, minimizando assim os efeitos nocivos das radiações e garantindo o destino final dos materiais infectados. O objetivo principal da gestão de rejeitos radioativos é garantir a proteção do homem e a preservação do meio ambiente quanto a radioatividade existente nesses rejeitos. O regulamento que estabelece as bases para a boa gestão dos rejeitos radioativos foi elaborado pela Comissão Nacional de Energia Nuclear, em 1985. Trata-se da Norma CNEN-NE-6.05: “Gerência de rejeitos radioativos em instalações radiativas”, que embora seja um marco relacionado a gestão dos rejeitos radioativos e ajude em grande parte no papel de orientar no projeto de um sistema de gestão em instalações radioativas de usuários de radioisótopos, aborda os tópicos de forma generalizada e não considera aspectos particulares das diferentes instalações, como é o caso dos serviços de medicina nuclear. O objetivo principal deste estudo é mostrar de forma simples e eficaz, a segregação e o acondicionamento seguro, evitando exposições desnecessárias de profissionais envolvidos, de pessoas que trabalham em áreas correlatas e dos indivíduos do público em geral.

Palavras-chave: Proteção Radiológica, Medicina Nuclear, Radioatividade

\section{INTRODUÇÃO}

A iodoterapia, embora utilizada a cerca de 50 anos, é uma terapêutica pouco divulgada, sendo capaz de tratar desde patologias benignas com alterações da função tireoidiana até patologias neoplásicas, tanto em adultos como em crianças (MATEUS, 2000). 
A iodoterapia é uma terapia complementar aplicada em sequência ao procedimento cirúrgico da retirada da tireoide que utiliza os isótopos radioativos do I-123 e I-131. A dose ministrada no tratamento de câncer de tireoide varia de $100 \mathrm{mCi}$ - para tecidos remanescentes depois da cirurgia - até $250 \mathrm{mCi}$ ou mais - para tratamento de metástases. O iodeto ou I-131 é poderoso no combate ao câncer e metástases por emitir partículas beta $(\beta)$ e radiação gama $(\gamma)$.

Segundo a CNEN, quando o paciente recebe dose acima de $30 \mathrm{mCi}$ de I-131, ele deve permanecer internado em unidades hospitalares, com acomodações que atendam a norma NE3.05. No período de internação, o paciente é atendido por uma equipe qualificada quanto ao conhecimento e aplicabilidade das normas de proteção radiológica do CNEN.

De acordo com Thompson (2001), após 24 horas da administração terapêutica do I-131, 35\% a 75\% são eliminados pela urina, suor e saliva. Apesar de pequena a porcentagem de rejeito radioativo eliminado pelo suor e saliva, estes são suficientes para contaminar o ambiente em que ele se encontra. Em consequência a isso, tornam-se necessários a utilização de equipamentos de proteção individual e comuns para os profissionais que trabalham diretamente com esse paciente, além de que todos os objetos utilizados no período de internação - vestimentas pessoais, roupas de cama, roupas de banho, copo, prato, talheres, restos alimentares - são considerados como contaminados, devendo ser tratados como rejeitos radioativos.

Os rejeitos radioativos gerados devem ser segregados e de acordo com a natureza física do material (sólido, líquido ou gasoso) e etiquetados de acordo com o radionuclídeo presente (identificação do tipo de amostra, procedência, data prevista para a liberação e símbolo do material radioativo). O material deve ser colocado em recipientes adequados, datados e mantidos no local da instalação destinado ao armazenamento provisório de rejeitos para aguardar o decaimento de forma segura.

Para o iodeto 131I o tempo estimado para decaimento é de cerca de 60 dias, podendo ocorrer antes, tornando necessário que o supervisor monitore semanalmente seus rejeitos no deposito. Para que ocorra a liberação do rejeitos, as amostras precisam apresentar taxa de exposição abaixo de $1 \mathrm{mR} / \mathrm{h}$. Após sofrer o processo de decaimento, os rejeitos se tornam-se lixo hospitalar comum, podendo ser encaminhados a aterros sanitários. 
O atual estudo foi realizado em um hospital de referência em Recife - Pernambuco, que dispõe do serviço de medicina nuclear. A pesquisa durou em média quatro meses e foi realizada no setor de iodoterapia da instituição. Durante esse período, todas as etapas do gerenciamento de rejeitos radioativos foram observadas e estudas de perto pelos pesquisadores com a finalidade de saber se este hospital segue as normas de radioproteção do durante todas as etapas do gerenciamento de rejeitos radioativos do órgão regulador (CNEN).

\section{MATERIAIS E MÉTODOS}

A pesquisa foi realizada com o auxílio de um detector cintilador do tipo Geiger Muller, (Imagem 1), que tem a função de capturar a taxa de exposição emitida por materiais infectados por substâncias radioativas. Um carrinho de transporte blindado, (Imagem 2), utilizado para conduzir os resíduos até o depósito.

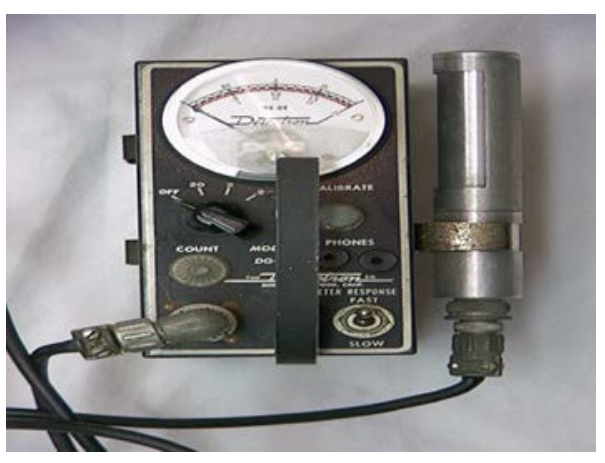

Imagem 1: detector Geiger Muller

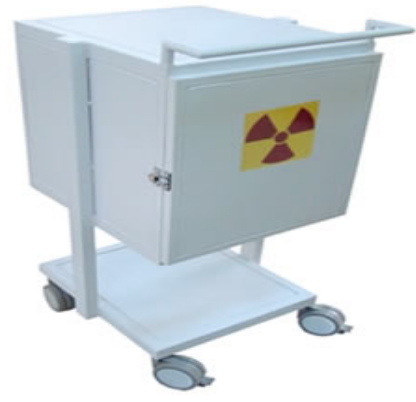

Imagem 2: carrinho de transporte blindado

Durante aproximadamente 120 dias, foram realizados levantamentos radiométricos entre os rejeitos gerados num determinado hospital, para a verificação da taxa de exposição e, aplicabilidade dos procedimentos de segurança na manipulação e acondicionamento de rejeitos radioativos. Os rejeitos eram recolhidos duas vezes por semana, segunda-feira e quarta-feira, sempre sucedendo uma internação. Após o quarto ser esvaziado pelo paciente que passou pelo tratamento, a equipe profissional recolheu todo o material utilizado pelo paciente durante sua estadia no hospital, e efetuou o levantamento radiométrico nas peças e objetos, para que fossem separados os materiais infectados com taxa superior a $1 \mathrm{mR} / \mathrm{h}$, e estes, postos em sacolas contendo o símbolo internacional das radiações e conduzidos, através do carrinho blindado, para o depósito de rejeitos.

As conferências de verificação de decaimento foram feitas nas sextas-feiras, sempre respeitando os limites permitidos pelas normas da CNEN, que diz que abaixo de $1 \mathrm{mR} / \mathrm{h}$ os rejeitos serão considerados lixo comum, devendo ser conduzidos para o aterro sanitário. 


\section{RESULTADOS E DISCUSSÕES}

O presente estudo teve como resultado os seguintes números:

Tabela 1: Levantamento radiométrico

\begin{tabular}{|c|c|c|c|c|c|c|}
\hline Material & $\begin{array}{l}\text { Data de } \\
\text { entrada }\end{array}$ & $\begin{array}{c}\text { Taxa na } \\
\text { superfície }\end{array}$ & $\begin{array}{l}\text { Taxa a um } \\
\text { metro }\end{array}$ & Peso & $\begin{array}{c}\text { Data de } \\
\text { saída }\end{array}$ & $\begin{array}{c}\text { Taxa de } \\
\text { saída }\end{array}$ \\
\hline Rejeito I - 131 & $23 / 09 / 13$ & $15,0 \mathrm{mR} / \mathrm{h}$ & $3,0 \mathrm{mR} / \mathrm{h}$ & $3 \mathrm{~kg}$ & $25 / 10 / 13$ & $0,7 \mathrm{mR} / \mathrm{h}$ \\
\hline Rejeito I - 131 & 25/09/13 & $13,8 \mathrm{mR} / \mathrm{h}$ & $2,7 \mathrm{mR} / \mathrm{h}$ & $2,5 \mathrm{~kg}$ & $25 / 10 / 13$ & $0,8 \mathrm{mR} / \mathrm{h}$ \\
\hline Rejeito I - 131 & $30 / 09 / 13$ & $6,0 \mathrm{mR} / \mathrm{h}$ & $1,4 \mathrm{mR} / \mathrm{h}$ & $1,6 \mathrm{~kg}$ & $01 / 11 / 13$ & $0,5 \mathrm{mR} / \mathrm{h}$ \\
\hline Rejeito I - 131 & $02 / 10 / 13$ & $9,0 \mathrm{mR} / \mathrm{h}$ & $1,8 \mathrm{mR} / \mathrm{h}$ & $1,3 \mathrm{~kg}$ & $01 / 11 / 13$ & $0,5 \mathrm{mR} / \mathrm{h}$ \\
\hline Rejeito I - 131 & 07/10/13 & $12,4 \mathrm{mR} / \mathrm{h}$ & $2,5 \mathrm{mR} / \mathrm{h}$ & $2,4 \mathrm{~kg}$ & 08/11/13 & $0,8 \mathrm{mR} / \mathrm{h}$ \\
\hline Rejeito I - 131 & 09/10/13 & $8,5 \mathrm{mR} / \mathrm{h}$ & $1,7 \mathrm{mR} / \mathrm{h}$ & $1,7 \mathrm{~kg}$ & $08 / 11 / 13$ & $0,6 \mathrm{mR} / \mathrm{h}$ \\
\hline Rejeito I - 131 & $14 / 10 / 13$ & $14,5 \mathrm{mR} / \mathrm{h}$ & $2,8 \mathrm{mR} / \mathrm{h}$ & $3,2 \mathrm{~kg}$ & $15 / 11 / 13$ & $0,8 \mathrm{mR} / \mathrm{h}$ \\
\hline Rejeito I - 131 & $16 / 10 / 13$ & $9,0 \mathrm{mR} / \mathrm{h}$ & $1,6 \mathrm{mR} / \mathrm{h}$ & $2,2 \mathrm{~kg}$ & 29/11/13 & $0,4 \mathrm{mR} / \mathrm{h}$ \\
\hline Rejeito I - 131 & $21 / 10 / 13$ & $10,8 \mathrm{mR} / \mathrm{h}$ & $2,3 \mathrm{mR} / \mathrm{h}$ & $1,8 \mathrm{~kg}$ & $22 / 11 / 13$ & $0,6 \mathrm{mR} / \mathrm{h}$ \\
\hline Rejeito I - 131 & 23/10/13 & $6,5 \mathrm{mR} / \mathrm{h}$ & $1,3 \mathrm{mR} / \mathrm{h}$ & $1,6 \mathrm{~kg}$ & 29/11/13 & $0,3 \mathrm{mR} / \mathrm{h}$ \\
\hline Rejeito I - 131 & 28/10/13 & $13,4 \mathrm{mR} / \mathrm{h}$ & $2,6 \mathrm{mR} / \mathrm{h}$ & $3,3 \mathrm{~kg}$ & 13/12/13 & $0,9 \mathrm{mR} / \mathrm{h}$ \\
\hline Rejeito I - 131 & $30 / 10 / 13$ & $8,7 \mathrm{mR} / \mathrm{h}$ & $1,6 \mathrm{mR} / \mathrm{h}$ & $2,4 \mathrm{~kg}$ & $06 / 12 / 13$ & $0,5 \mathrm{mR} / \mathrm{h}$ \\
\hline Rejeito I - 131 & $04 / 11 / 13$ & $18,4 \mathrm{mR} / \mathrm{h}$ & $3,7 \mathrm{mR} / \mathrm{h}$ & $3,8 \mathrm{~kg}$ & 20/12/13 & $0,8 \mathrm{mR} / \mathrm{h}$ \\
\hline Rejeito I - 131 & $06 / 11 / 13$ & $6,0 \mathrm{mR} / \mathrm{h}$ & $1,2 \mathrm{mR} / \mathrm{h}$ & $1,8 \mathrm{~kg}$ & $06 / 12 / 13$ & $0,3 \mathrm{mR} / \mathrm{h}$ \\
\hline Rejeito I - 131 & $11 / 11 / 13$ & $13,5 \mathrm{mR} / \mathrm{h}$ & $2,6 \mathrm{mR} / \mathrm{h}$ & $2,7 \mathrm{~kg}$ & $20 / 12 / 13$ & $0,7 \mathrm{mR} / \mathrm{h}$ \\
\hline Rejeito I - 131 & $13 / 11 / 13$ & $8,8 \mathrm{mR} / \mathrm{h}$ & $1,7 \mathrm{mR} / \mathrm{h}$ & $2,1 \mathrm{~kg}$ & $06 / 12 / 13$ & $0,4 \mathrm{mR} / \mathrm{h}$ \\
\hline Rejeito I - 131 & $18 / 11 / 13$ & $16,0 \mathrm{mR} / \mathrm{h}$ & $3,2 \mathrm{mR} / \mathrm{h}$ & $2,6 \mathrm{~kg}$ & $17 / 01 / 14$ & $0,8 \mathrm{mR} / \mathrm{h}$ \\
\hline Rejeito I - 131 & 20/11/13 & $9,6 \mathrm{mR} / \mathrm{h}$ & $1,8 \mathrm{mR} / \mathrm{h}$ & $1,8 \mathrm{~kg}$ & 20/12/13 & $0,4 \mathrm{mR} / \mathrm{h}$ \\
\hline Rejeito I - 131 & 25/11/13 & $13,0 \mathrm{mR} / \mathrm{h}$ & $2,6 \mathrm{mR} / \mathrm{h}$ & $2,4 \mathrm{~kg}$ & $17 / 01 / 14$ & $0,7 \mathrm{mR} / \mathrm{h}$ \\
\hline Rejeito I - 131 & $27 / 11 / 13$ & $7,5 \mathrm{mR} / \mathrm{h}$ & $1,3 \mathrm{mR} / \mathrm{h}$ & $1,9 \mathrm{~kg}$ & 20/12/13 & $0,3 \mathrm{mR} / \mathrm{h}$ \\
\hline
\end{tabular}

Os materiais recolhidos têm um tempo estimado de quatro meses para o decaimento radioativo, segundo normas da CNEN, pois trata-se de contágio por I - 131, que tem um tempo de meia-vida de 8,4 dias. As doses entre 250 e $400 \mathrm{mCi}$, foram efetuadas sempre nos internamentos da quinta-feira, sendo o rejeito coletado na segunda-feira posterior ao tratamento. Pudemos constatar que quanto maior a dose no paciente, maior será a taxa de exposição dos rejeitos, uma vez que este material radioativo é eliminado por vias de excretas e contamina totalmente todos os materiais usados pelos pacientes no período de internamento. Os outros pacientes, internados na quarta-feira, recebiam doses entre 100 e $200 \mathrm{mCi}$, necessitando apenas de 24 a 36 horas de internamento, pois para receber alta, o paciente precisa estar com taxa de exposição inferior a $5 \mathrm{mR} / \mathrm{h}$. Já os pacientes da quinta-feira, ficaram internados até o sábado, devido as altas doses aplicadas para tratamento.

Observamos também que houve uma queda brusca no tempo de decaimento do material infectado, em relação a norma vigente. Os materiais passaram em média dois meses 
acondicionados no galpão destinado para rejeitos, sendo monitorados semanalmente, para a verificação das taxas, e seu possível encaminhamento para o aterro sanitário.

O trajeto feito pela equipe, para levar os rejeitos radioativos até o deposito, durou em média 5 minutos, com taxa média de exposição de $3,0 \mathrm{mR} / \mathrm{h}$, a um metro da região geniturinária. Foram utilizados capotes de $0,5 \mathrm{~mm}$ de chumbo por toda equipe, para minimizar as ações das radiações ionizantes.

\section{CONCLUSÕES}

A iodoterapia é uma terapia complementar, pós-cirurgia, que visa o tratamento de patologias da tireoide, seja para ablação de tecidos remanescentes, ou tratamento de metástases, fazendo uso do I-131. Esse radioisótopo apresenta energia de $364 \mathrm{Kev}$ e tempo de meia-vida de 8,4 dias. Por sua energia ser eliminada principalmente pelo suor, saliva e urina, é necessária uma atenção maior para os materiais utilizados durante o tratamento, bem como roupas, produtos de higiene pessoal, objetos pessoais. Durante o tratamento uma equipe especializada e devidamente familiarizada com as normas de proteção da lei vigente permanecerá cuidando e monitorando o paciente, que só poderá receber alta quando a atividade presente de I-131 for igual ou inferior a $5 \mathrm{mR} / \mathrm{h}$ segundo normas da CNEN. Após o internamento, todos os utensílios utilizados pelo paciente deverão ser tratados como rejeitos radioativos merecendo atenção especial. É da responsabilidade do hospital segregar e armazenar esse material em lugares adequados até que a amostra decaia o suficiente para ser encaminhada a um depósito de decaimento ou aterro sanitário de acordo com as especificações do órgão fiscalizador.

É de extrema importância a observância de todos os procedimentos abordados neste estudo, bem como o cumprimento de todas as exigências das normas vigente visando sempre a prevenção de acidentes e exposições desnecessárias, tanto de trabalhadores, como do público.

\section{REFERÊNCIAS}

1. Journal

CALEGARO, J.U.M., TEIXEIRA, S.M.P., Avaliação da exposição ocupacional de auxiliares de enfermagem na iodoterapia durante 11 anos. Radiologia Brasileira, v.40, p. 263-266, 2007. 
CUSSIOL, N.A.M, SILVA, E.M.P., Gerência de rejeitos radioativos de serviços de saúde. Comissão Nacional de Energia Nuclear e Centro de Desenvolvimento da Tecnologia Nuclear., v. 35, n.5 Belo Horizonte, Publicação CDNT-857/99.

MATEUS, L., A importância da Enfermagem no Tratamento com Iodo Radioativo. Revista Nursing. n. 25, p.6-8, 2000.

THOMPSON, M.A., Radiation Safety Precautions in the Manegement of the Hospitalized 131I Therapy Patient. Journal of Nuclear Medicine Technology, v. 29, n.2, 2001.

\section{Book}

CASTRO, A. Jr. et al., Guia prático em medicina nuclear a instrumentação, $2^{\text {nd }}$ ed. São Paulo: SENAC, 2000.

\section{Report}

CONSELHO NACIONAL DE ENERGIA NUCLEAR. Norma de Requisitos de Radioproteção e Segurança para Serviços de Medicina Nuclear Norma de Diretrizes Básicas de Proteção Radiológica - NE-3.01, CNEN, $01 / 2005$.

Norma de Requisitos de Radioproteção e Segurança para Serviços de Medicina Nuclear - NE-3.05, CNEN, 03/1996. . Norma de Gerência de Rejeitos Radioativos em Instalações Radioativas NE-6.05, CNEN, 10/1996.

\section{Web site}

ALMEIDA, A.T.Jr. "Caracterização da barita e do vidro cristal como atenuadores na blindagem das radiações X e GAMA”. 2005, 53 p. Dissertação (Mestrado em Energia Nuclear) - Universidade Federal de Pernambuco, Recife. Available at: <http://www.fundacentro.gov.br/CTN/teses_pdf/dissertação_AIRTON.pdf> Last accessed: 3 Jul. 2014 
André R. M. Silva, et. al. • Braz. J. Rad. Sci. • 2015

Instituto de Radioproteção e Dosimetria. “O que são rejeitos radioativos?”. Available at: $<$ http://www.ird.gov.br/index.php?option=com_content\&view=article\&id=60>

Last accessed: 5 Jul. 2014 